\title{
DESENVOLVIMENTO E IMPLANTAÇÃO DA LIGA ACADÊMICA DE FUNCIONALIDADE HUMANA: RELATO DE EXPERIÊNCIA
}

\section{DEVELOPMENT AND IMPLEMENTATION OF THE ACADEMIC LEAGUE OF HUMAN FUNCTIONALITY: EXPERIENCE REPORT}

\section{DESARROLLO E IMPLEMENTACIÓN DE LA LIGA ACADÉMICA DE FUNCIONALIDAD HUMANA: UN INFORME DE EXPERIENCIA}

Giulianna de Brito Brasil' ${ }^{1}$, Francisca Andressa Teixeira de Oliveira' ${ }^{1}$, Paulo Ricardo Silva Lopes ${ }^{1}$, Brena Caroline de Sousa Monteiro', Bruna Loureiro Lô', Joyce Moreira Souza1', Kleyce Fernanda Prestes Carneiro', Roger Berg Rodrigues Pereira1, Luara de Alencar Sousa', Maria Valcilene Leite ${ }^{1}$, Thiago Brasileiro de Vasconcelos ${ }^{1}$

Submetido: 10/06/2020

Aprovado: 25/09/2020

\section{RESUMO}

As Ligas Acadêmicas são entidades criadas por acadêmicos, professores e profissionais que apresentam interesse em determinado tema. Realizam atividades extraclasse, com ações voltadas para a educação, e os temas abordados são relacionados ao que é estudado na sala de aula. O presente relatou a experiência da criação da Liga Acadêmica de Funcionalidade Humana (LAFUN) por estudantes do curso de Fisioterapia da Uninassau - Parangaba. Foi realizado um estudo do tipo relato de experiência de caráter qualitativo, desenvolvido por estudantes e docentes que fazem parte da LAFUN, escolhendo como tema principal a funcionalidade humana. A liga foi criada com a intenção de proporcionar a importância de estudar, pesquisar e promover a funcionalidade humana nos diferentes perfis populacionais, e ademais, abordar a relação saúde e doença de acordo com o ambiente em que estão inseridos. Conclui-se que a criação e implementação da Liga Acadêmica de Funcionalidade Humana proporcionou um avanço no conhecimento e atuação docente/discente relacionado à funcionalidade humana.

PALAVRAS-CHAVE: Funcionalidade; Fisioterapia; Educação Superior

\begin{abstract}
Academic Leagues are entities created by academics, teachers and professionals who have an interest in a topic. They carry out extra-class activities, with actions aimed at education, and the topics covered are related to what is studied in the classroom. The present reported the experience of the creation of the Academic League of Human Functionality (LAFUN) by students of the Physiotherapy course at Uninassau - Parangaba. A qualitative experience report study was carried out, developed by students and teachers who are part of LAFUN, choosing human functionality as the main theme. The league was created with the intention of providing the importance of studying, researching, and promoting human functionality in different population profiles, and in addition, addressing the relationship between health and disease according to the environment in which they operate. It is concluded that the creation and implementation of the Academic League of Human Functionality provided an advance in knowledge and teaching/ student performance related to human functionality.
\end{abstract}

KEYWORDS: Functionality; Physiotherapy; College education

${ }^{1}$ Liga Acadêmica de Funcionalidade Humana (LAFUN). Centro Universitário Uninassau - Parangaba, Fortaleza, Ceará, Brasil. 


\section{RESUMEN}

Las ligas académicas son entidades creadas por académicos, profesores y profesionales que tienen interés en un tema en particular. Llevan a cabo actividades extra-clase, con acciones dirigidas a la educación, y los temas cubiertos están relacionados con lo que se estudia en el aula. El presente informó sobre la experiencia de la creación de la Liga Académica de la Funcionalidad Humana (LAFUN) por parte de los estudiantes del curso de Fisioterapia en Uninassau Parangaba. Se realizó un estudio de informe de experiencia cualitativa, desarrollado por estudiantes y profesores que forman parte de LAFUN, eligiendo la funcionalidad humana como tema principal. La liga fue creada con la intención de proporcionar la importancia de estudiar, investigar y promover la funcionalidad humana en diferentes perfiles de población y, además, abordar la relación entre la salud y la enfermedad según el entorno en el que operan. Se concluye que la creación e implementación de la Liga Académica de Funcionalidad Humana proporcionó un avance en el conocimiento y el desempeño docente / estudiantil relacionado con la funcionalidad humana.

PALABRAS CLAVE: Funcionalidad; Fisioterapia; Educación universitaria 


\section{INTRODUÇÃO}

As Ligas Acadêmicas (LA) foram criadas na época da ditadura militar - a primeira em 1920 Liga de Combate a Sífilis -, época que ascendeu questionamentos sobre o ensino propagado nas universidades'1.

São definidas como entidades formadas por grupos de alunos de diferentes anos de graduação sob supervisão de profissionais e professores vinculados a Instituição de Ensino Superior ou Hospitais de Ensino². Não tem fins lucrativos, é apartidária, não religiosa, tendo duração ilimitada e com carácter multiprofissional, com o objetivo de incentivar o estudo de um determinado assunto, projetos científicos e atividade assistencial voluntária à comunidade ${ }^{3}$.

Atualmente, as Ligas Acadêmicas possuem caráter relevante, já que as instituições de ensino superior vêm se transformando ao longo dos anos, no entanto, tais transformações não conseguem ser difundidas na sociedade4.

Em consonância, o Conselho Nacional de Educação destaca que entre as competências e habilidades gerais requeridas para a formação do Fisioterapeuta, destaca-se:

“Educação permanente: os profissionais devem ser capazes de aprender continuamente, tanto na sua formação, quanto na sua prática. Desta forma, os profissionais de saúde devem aprender a aprender e ter responsabilidade e compromisso com a sua educação e o treinamento/estágios das futuras gerações de profissionais, mas proporcionando condições para que haja benefício mútuo entre os futuros profissionais e os profissionais dos serviços, inclusive, estimulando e desenvolvendo a mobilidade acadêmico/profissional, a formação e a cooperação através de redes nacionais e internacionais. Competências e Habilidades Específicas: atuar multiprofissionalmente, interdisciplinarmente e transdisciplinarmente com extrema produtividade na promoção da saúde baseado na convicção científica, de cidadania e de ética" 5 .

Uma Liga Acadêmica é formada por um grupo de alunos e professores com um interesse comum, que se reúnem para realizar atividades práticas e teóricas sobre um tema, supervisionados por um ou mais profissionais da área6.

Sendo assim, ela atua como ferramenta para a consolidação do aprendizado das habilidades teórico-práticas na área de funcionalidade humana, para tanto, utiliza como recurso aulas teóricas (grupo de estudo, apresentação de casos clínicos, palestras), aulas práticas (contato direto com pacientes), jornadas acadêmicas, pesquisas na área de atuação da liga, trabalhos de extensão, estágios supervisionados nos hospitais e reuniões para o planejamento das atividades durante $\mathrm{o}$ ano.

Para se destacar no mercado de trabalho, os universitários precisam ter um diferencial, algo especial que possa complementar a sua formação acadêmica e o aprendizado das salas de aulas. As atividades extracurriculares são fundamentais nesse aspecto, e cada estudante se direciona para aquilo que mais lhe interessa $e$ Ihe agrega conhecimentos. Entre as diversas possibilidades, as ligas acadêmicas são uma ótima oportunidade para quem deseja aprender mais sobre determinada área do seu campo de formação.

Sendo assim, o presente estudo pretende relatar a experiência da criação da Liga Acadêmica de Funcionalidade Humana (LAFUN) por estudantes do curso de Fisioterapia da Uninassau Parangaba.

\section{MÉTODO}

Trata-se de um estudo do tipo relato de experiência relacionado ao processo de ensino-aprendizagem em Fisioterapia, de abordagem qualitativa, interpretativa através da descrição das fases de planejamento, implantação e execução da Liga Acadêmica de Funcionalidade Humana, ocorrido em 2018, e sua operacionalização, em 2019.

A escolha por uma pesquisa qualitativa se deu por ela não se apresentar como uma proposta rigidamente estruturada, permitindo o uso da imaginação e da criatividade levando a propostas que enfoquem novas abordagens7.

Nesse contexto, a pesquisa abordada no presente estudo busca sanar duas vertentes principais: um lado a sensibilidade à interpretação dos dados e por outro as concepções da experiência humana, ou seja, o significado é construído socialmente8-9.

O relato de experiência destaca-se como um texto que descreve com máximo de precisão um fato que pode contribuir para a sua área de atuação. Sendo assim, os autores descrevem uma vivência profissional exitosa ou não que pode contribuir para a discussão, 
troca de experiências e propostas de ideias para a meIhoria do cuidado10.

Durante a elaboração e implantação os autores atuaram como participantes e observadores. Este trabalho foi desenvolvido por estudantes e docentes que fazem parte da Liga Acadêmica de Funcionalidade Humana (LAFUN), criada em 2018 através da aprovação pela Coordenação do Curso de Fisioterapia da Uninassau - Sede Parangaba, Fortaleza/CE.

A Liga tem como objetivo o tripé do Ensino Superior - desenvolver atividades de ensino, a pesquisa e extensão. A funcionalidade foi o tema central para a criação da liga, já que o estudo da funcionalidade é objeto de interesse em diversos públicos, dentre eles: idosos, adolescentes, crianças, entre outros; possibilitando assim, uma gama de abordagens que podem ser realizadas através da liga, ademais, ressalta-se que a referente liga é pioneira com relação a esta temática.

\section{RELATO DE EXPERIÊNCIA}

A liga acadêmica de funcionalidade humana foi criada com o interesse em pesquisas, estudar casos de fisioterapia voltados para o conceito da Classificação Internacional de Funcionalidade (CIF) e o desenvolvimento de atividades acadêmicas científicas relacionados à área.

A criação da liga partiu do interesse de alguns estudantes que encaminharam a ideia para um docente de instituição e o mesmo organizou a documentação da liga e o processo seletivo, composto de prova teórica de múltipla escolha e entrevista, sendo aprovado 10 alunos. Todos os alunos participantes da liga estão cursando entre o $3^{\circ}$ e $7^{\circ}$ semestre do curso de fisioterapia.

Na primeira reunião, que ocorreu na sede da Uninassau - Parangaba, ficou acordado que haveria reuniões quinzenais que seriam registradas no livro ATA, onde o coordenador e todos os membros assinaram como forma de presença e participação. O coordenador apresentou o Estatuto da Liga Acadêmica e sua finalidade, determinou a função específica de cada membro, e que foi de comum acordo para cada um dos participantes.

\footnotetext{
A Liga tem por finalidades:

I. Despertar o interesse pelo estudo de temas relevantes funcionalidade humana.

II. Iniciar e propiciar o desenvolvimento da vivência teórico-prática na área de funcionalidade humana aos alunos graduandos.

III. Organizar e auxiliar na promoção de ações
}

de caráter científico e social que visem o aprimoramento da formação acadêmica.

IV. Observação e estudo de casos acompanhados na clínica de fisioterapia, registro dos casos observados, divulgação das informações com os membros desde que não seja divulgada a identidade do paciente e que não fira a ética e a bioética.

V. Proporcionar aos membros efetivos e coordenação a possibilidade de participação em projetos de pesquisa.

VI. Estimular o acesso às publicações científicas da área de funcionalidade humana aplicada a área da saúde com ênfase na análise crítica a partir de reuniões periódicas.

VII. Desenvolver grupos de estudo e discussões, palestras, mesas redondas, simpósios e cursos relacionados aos interesses da Liga. VIII. Fornecer informes de cursos e congressos na área de funcionalidade humana.

IX. Organizar e participar de cursos, palestras, jornadas, congressos, simpósios e outras atividades cientificas.

(Art. 40: Estatuto da LAFUN).

\section{- Organograma LAFUN:}

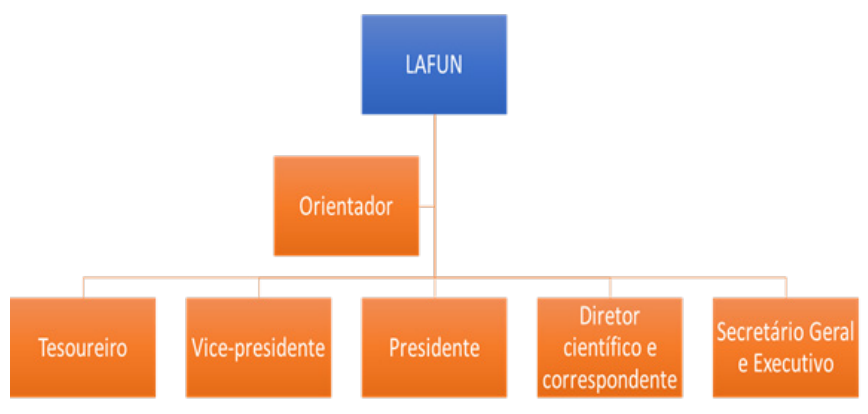

Cabe ao Presidente: Aluno 1 - Representar a LAFUN junto aos vários órgãos da Faculdade Maurício de Nassau e à comunidade; Coordenação geral da Liga, presidir as reuniões da Diretoria e das Assembleias Gerais e supervisionar todos os projetos desenvolvidos pela liga; Convocar as reuniões extraordinárias; Assinar juntamente com o(s) secretário(s) as atas e outros documentos da Liga; Reunir-se com instituições públicas e privadas, a fim de obter recursos e/ou objetos e produtos necessários para a execução das atividades da Liga.

Vice-presidente: Aluno 2 - Substituir, com as mesmas atribuições, o presidente, nos casos de ausência ou impedimento deste; Auxiliar o presidente em todas 
as suas funções e auxiliar nas funções das demais atribuições.

Secretários Geral e Executivo: Alunos 3, 4 e 5 - Movimentar a correspondência da LAFUN; Secretariar as reuniões da Diretoria e das Assembleias Gerais com registro em ata; Controlar o número de faltas dos membros nas atividades obrigatórias; Apresentar mensalmente o balanço das atividades teóricas e práticas da LAFUN à Diretoria e à Assembleia Geral Ordinária; Elaborar todas as formas de divulgação da LAFUN.

Tesoureiro: Aluno 6 - Efetuar transações financeiras relacionadas às atividades da LAFUN; Envolver-se na busca de colaboração financeira junto às instituições interessadas.

Diretor Científico e correspondentes: Alunos 7, 8, 9 - Incentivar as pesquisas científicas nas diversas áreas; organizar outras atividades científicas da LAFUN; organizar curso anual preparatório para prova de admissão da LAFUN; Organizar os acervos bibliográficos pertencentes à liga; Manter os membros da liga atualizados sobre Congressos, Jornadas e outras atividades referentes à área da liga.

Da Supervisão: Aluno 10 - Orientar e avaliar as atividades propostas, promovidas e executadas pela liga; orientar a Diretoria em atribuições relacionadas a esta.

Com todos os membros cientes de suas atribuições, começamos a pensar e elaborar um símbolo (logomarca) e uma blusa para representar a liga acadêmica, um e-mail e um grupo de conversas no WhatsApp $\AA$ para facilitar a comunicação e marcação de reuniões. Criamos também uma página no Instagram $®$ (@lafun_parangaba) com intuito de divulgarmos conteúdos pertinentes a funcionalidade e bem-estar no âmbito da fisioterapia ao público, além de divulgar as atividades realizadas pela liga ou pela Instituição.

Organizamos o primeiro evento científico, intitulado como primeiro Circuito Saúde (Figura 1), no qual convidamos alguns fisioterapeutas, das mais variadas áreas de atuação, para abordar alguns temas, ministrados em forma de palestra. O evento ocorreu no auditório da sede Uninassau - Parangaba, foi bem divulgado e contamos com a presença de muitos estudantes na área da saúde.
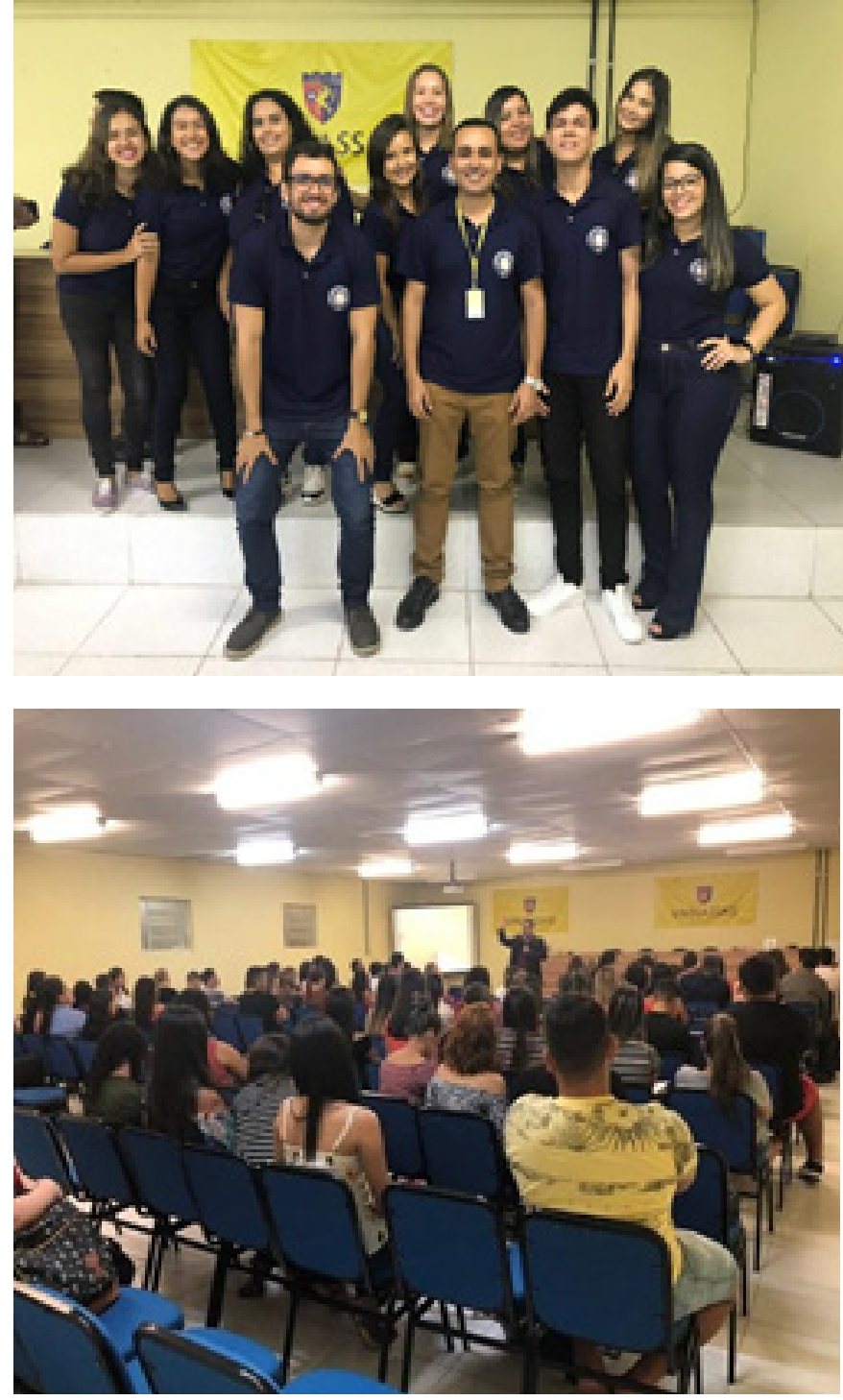

Figura 1. Imagens da Organização do $1^{\circ}$ Circuito Saúde Uninassau Parangaba.

Legenda: $\mathrm{A}$ imagem à esquerda representa todos os integrantes da LAFUN. A imagem à direita representa o Auditório e o público composto por acadêmicos de fisioterapia de diferentes semestres.

Nas nossas reuniões observamos a necessidade de uma ação educativa nas academias com enfoque de postura correta, prevenção de lesões e dentre outras técnicas de terapia manual (p. ex. liberação miofascial, inibição muscular) e ventosaterapia (Figura 2).

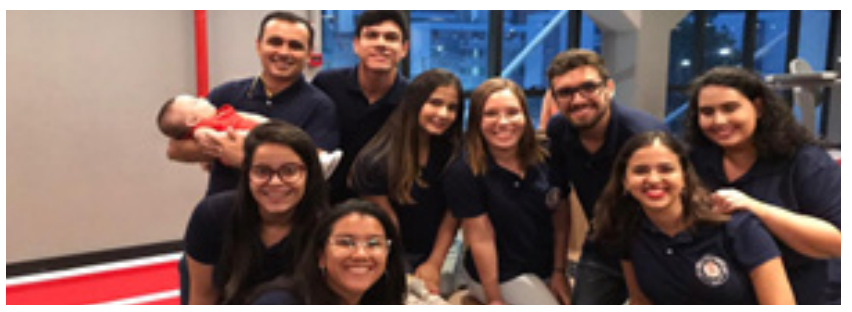




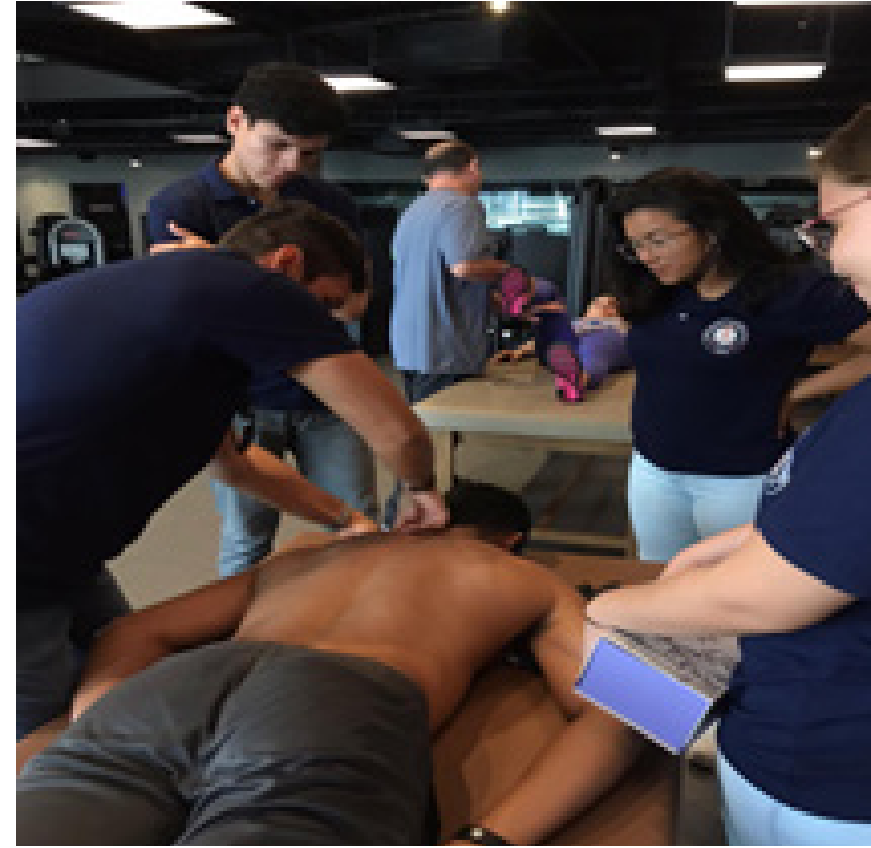

Figura 2. Imagens da atividade na academia.

Realizamos apresentações de trabalhos em eventos científicos, destacamos a apresentação do trabaIho intitulado: Exercícios físicos associados a gestação (uma revisão bibliográfica) na Escola de Saúde Pública do Ceará (Figura 3).

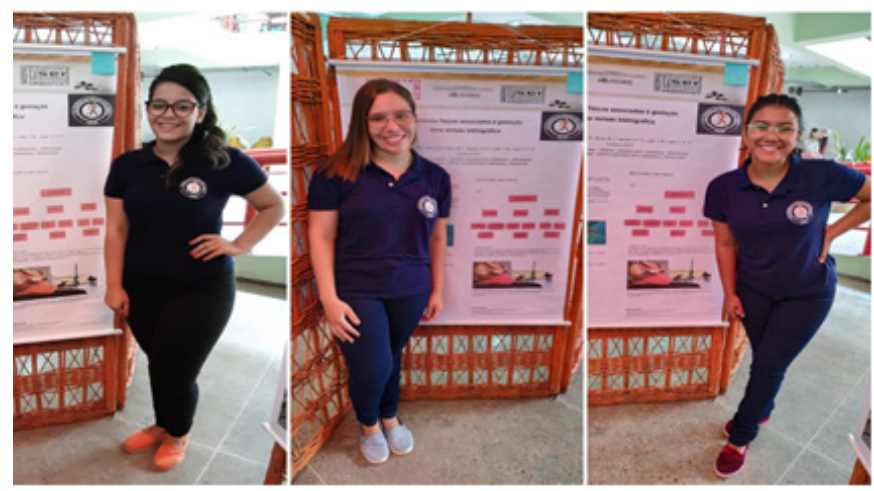

Figura 3. Apresentação do trabalho no formato do banner.

No mês da mulher realizamos uma atividade em um shopping center na cidade de Fortaleza (Figura 4), onde elaboramos um panfleto educativo e ilustrativo sobre cuidados na saúde da mulher e o autoexame das mamas.

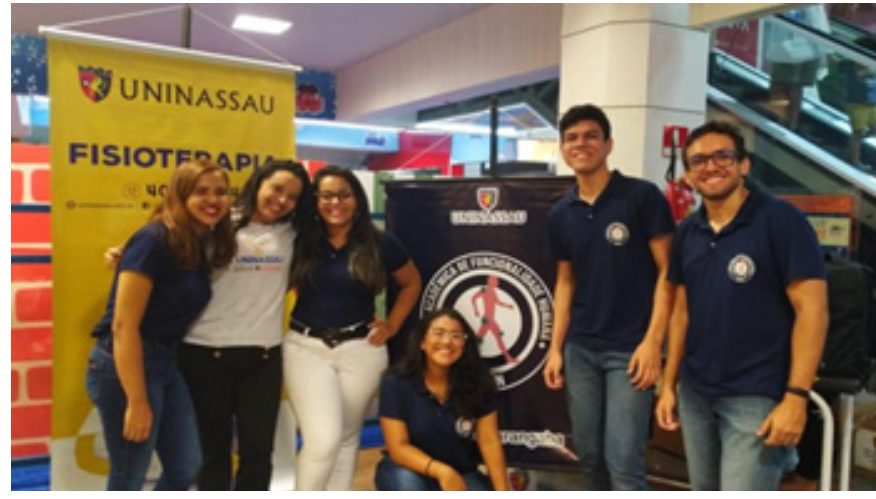

Figura 4. Ação sobre a Saúde da Mulher.

\section{DISCUSSÃO}

De acordo com Sampaio e Luz11, a Organização Mundial da Saúde (OMS) aprovou em 2001 a Classificação Internacional de Funcionalidade, Incapacidade e Saúde (CIF), a CIF trabalha com o modelo biopsicossocial que tenta abordar a integração entre o biológico, o social e o individual. A funcionalidade e a incapacidade humanas são concebidas como uma interação dinâmica entre as condições de saúde (doença, trauma, lesões, distúrbios) e os fatores contextuais (incluindo fatores pessoais e ambientais). Portanto, esta LA foi criada com a intenção de proporcionar a importância de estudar, pesquisar e promover a funcionalidade humana nos diferentes perfis populacionais, e ademais, abordar a relação saúde e doença de acordo com o ambiente em que estão inseridos.

As LA's que abordam o tema funcionalidade são muito diversas, e elas presam pela abordagem biopsicossocial. Em Porto Alegre, município no Rio Grande do Sul, existe a Faculdade de Desenvolvimento do Rio Grande do Sul (FADERGS) que trabalha com uma LA interdisciplinar voltada para a funcionalidade e dor. Nela ocorre o desenvolvimento de trabalhos de pesquisa, palestras e um convívio com a comunidade onde promovem ações, como: doação de roupas, conscientização sobre o câncer de mama e próstata, prevenção ao suicídio e a dor crônica12.

Silva e Flores13 afirmam que as LA conformariam um espaço demarcado pela intersecção entre práticas do sistema formador em Saúde, Sistema Único de Saúde (SUS) e a Rede de Serviços Públicos articulada a comunidade, mostrando assim que os alunos que participam dessas instituições têm um processo de educação e de profissionalização em saúde maior do que outros alunos, por estarem em contato com pacientes continuamente. 
O desempenho das LA resulta do interesse por parte dos seus participantes, pois uma liga engloba um ensino diferente do habitual acadêmico13. Torres ${ }^{1}$ afirma que as LA funcionam como um método de inserir os acadêmicos em atividades educativas diferenciadas, juntos ao seu orientador tendo como um dos objetivos uma maior interação entre os acadêmicos e a comunidade. Desta maneira, os participantes devem buscar métodos através da liga que facilitem essa interação com a população como pesquisas, campanhas, simpósios com a finalidade de prevenção e promoção à saúde da população, e uma maior amplitude de conhecimentos.

A LA na qual este estudo é baseado tem como princípios iniciais trabalhar dois temas principais em seus trabalhos acadêmicos, estes temas são áreas da fisioterapia nas quais seus membros tem mais afinidade, são eles Gerontologia e Traumato-Ortopédica. Para Aciole e Batista14, o envelhecimento em grande amplitude é uma realidade no mundo contemporâneo, e com esse avanço na faixa etária da população há novas mudanças em relação à morbidade e a mortalidade, pois há um aparecimento de doenças crônicas comuns que afetam a população idosa. No Brasil, a assistência aos idosos é uma realidade mais difícil de ser articulada, principalmente por conta do seu vasto crescimento.

Nogueira et al.15 afirma que a fisioterapia Traumato-Ortopédica é a área que mais ganha visibilidade, por causa dos distúrbios do sistema muscular, ósseo e articular que são a segunda busca de atendimento médico no país, além disso essas alterações acarretam ainda distúrbios funcionais que comprometem as atividades diárias do indivíduo.

Nesta perspectiva, Queiroz et al.16 afirma que é consenso a importância das Ligas Acadêmicas para o crescimento e desenvolvimento do aluno, com maior ênfase na aquisição do conhecimento, ampliação do senso crítico e raciocínio científico.

Não obstante, Cavalcante et al.17 afirma que ainda há uma precária literatura sobre as LA. De forma adicional, é importante pensar sobre como as LA estão atuando quanto extensão universitária, de modo a identificar e enfocar nos resultados das mesmas17-18.

É notório que as atividades de promoção e prevenção de doenças são de fundamental importância para a formação acadêmica, tendo como objetivo despertar o interesse discente sobre esta temática na busca de cerrar a lacuna existente entre a Atuação Fisioterapêutica e a Saúde Pública.

\section{CONCLUSÃO}

Evidenciou-se que a criação e implementação da Liga Acadêmica de Funcionalidade Humana proporcionou um avanço no conhecimento e atuação docente/ discente relacionado à esta temática.

\section{REFERÊNCIAS}

1. Torres AR. et al. Academic Leagues and medical education: contributions and challenges. Interface - Comunic Saúde Educ. 2008;12(27):713-20.

2. Monteiro LLF. et al. Ligas acadêmicas: o que há de positivo? Experiência de implantação da Liga Baiana de Cirurgia Plástica. Revista Brasileira de Cirurgia Plástica. 2008;23(3):158-61.

3. Costa AP. et al. A importância da Liga Acadêmica de Queimaduras. Revista Brasileira de Queimaduras. 2009;8(3):101-5.

4. Barbieri LG. et al. Liga Acadêmica de Fisioterapia em Ortopedia e Traumatologia no Esporte - LORTE: um relato de experiência. Rev Ciênc Ext. 2015;11(1):161-70.

5. Brasil. Conselho Nacional de Educação. Resolução CNE/CES 4/2002. Diário Oficial da União, Brasília 2002; Seção 1:11.

6. UNIVERSIDADE FEDERAL DE SÃO PAULO (UNIFESP). Programa de Orientação e Atendimento a Dependentes. Acesso em: 2020 maio 15. Disponível em: http://www.proad.unifesp.br/liga_faq.htm.

7. Godoy, AS. Pesquisa qualitativa: aspectos fundamentais. Revista RAE. 1995;25(3):20-29.

8. Moita-Lopes, LP. Pesquisa interpretativista em lingüística aplicada: a linguagem como condição e solução. DELTA. 1994.10(2):329-338.

9. Fontelles MJ, et al. Metodologia da pesquisa científica: diretrizes para a elaboração de um protocolo de pesquisa. Rev. para. med. 2009;23(3).

10. Universidade Federal de Juiz de Fora. Instrutivo para elaboração de relato de experiência: Estágio em Nutrição em Saúde Coletiva. Acesso em: 
2020 agosto 15. Disponível em: https://www.ufjf.br/ nutricaogv/files/2016/03/Orienta\%C3\%A7\%C3\%B5es-Elabora\%C3\%A7\%C3\%A3o-de-Relato-de-Experi\%C3\%AAncia.pdf.

11. Luz MT, Sampaio RF. Funcionalidade e incapacidade humana: explorando o escopo da classificação internacional da Organização Mundial da Saúde. Caderno Saúde Pública. 2009;25(3):475-483.

12. Faculdade de Desenvolvimento do Rio Grande do Sul (FADERGS). Liga Acadêmica Interdisciplinar de Estudos da Dor e Funcionalidade. Acesso em: 2020 agosto 15. Disponível em: https://www.fadergs.edu.br/ noticias/noticias/liga-academica-interdisciplinar-de-estudos-da-dor-e-funcionalidade-da-fadergs.

13. Flores O, Silva SA. Ligas Acadêmicas no Processo de Formação dos Estudantes. Revista Brasileira de Educação Médica. 2015;39(3):410-425.

14. Aciole GG, Batista LH. Promoção da saúde e prevenção de incapacidades funcionais dos idosos na estratégia de saúde da família: a contribuição da fisioterapia. Saúde em Debate. 2013; 37(96):10-19.

15. Nogueira AF, et al. Principais distúrbios Traumato-Ortopédicos atendidos em clínicas-escola de fisioterapia. Revista Científica da Faculdade de Educação e Meio Ambiente. 2016;7(2):33-44.

16. Queiroz SJ, et al. A Importância das ligas acadêmicas na formação profissional e promoção de saúde. Fragmentos de Cultura. 2014;24(especial):73-78.

17. Cavalcante ASP, et al. As ligas acadêmicas na área da saúde: lacunas do conhecimento na produção científica brasileira. RBEM. 1018;42(1):194-204.

18. Goergen DI. Ligas acadêmicas: uma revisão de várias experiências. Arq. Catarin Med. 2017;46(3):183193. 